Agronomía Costarricense 35(2): 21-32. ISSN:0377-9424 / 2011

www.mag.go.cr/rev_agr/index.html www.cia.ucr.ac.cr

\title{
EFECTO DEL VERMICOMPOST Y QUITINA SOBRE EL CONTROL DE Meloidogyne incognita EN TOMATE A NIVEL DE INVERNADERO
}

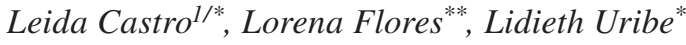

Palabras clave: Meloidogyne incognita, vermicompost, quitina, tomate, supresión de nematodos. Keywords: Meloidogyne incognita, vermicompost, chitin, tomato, nematodes suppression.

Recibido: 13/12/10

RESUMEN

Meloidogyne incognita es un nematodo endoparásito sedentario, de penetración total y formador de nódulos radicales; tiene una amplia distribución geográfica y causa pérdidas agrícolas importantes. Recientemente se han utilizado abonos orgánicos y compuestos como la quitina en la supresión de plagas y enfermedades de plantas. El objetivo de esta investigación fue evaluar el efecto de la aplicación de vermicompost de estiércol bovino y su enriquecimiento con quitina, sobre la infección de $M$. incognita en plantas de tomate var. Hayslip bajo condiciones de invernadero. Para esto se inocularon las plantas con 5000 unidades de inóculo (huevos+juveniles en segundo estado) por maceta y se compararon con controles que fueron sembrados en suelo sin enmienda orgánica o quitina. La evaluación se llevó a cabo 60 días después y se midió el peso fresco aéreo y radical, peso seco aéreo, población total de nematodos en raíz y suelo, índice de nódulos radicales (INR), índice de masas de huevos (IMH), el factor de reproducción del nematodo (FR) y recuento de grupos funcionales

1 Autor para correspondencia: Correo electrónico: leida.castro@ucr.ac.cr

* Centro de Investigaciones Agronómicas, Universidad de Costa Rica. San José, Costa Rica.
Aceptado: 02/06/11

\begin{abstract}
Effect of vermicompost and chitin on the control of Meloidogyne incognita in greenhouse tomato. Meloidogyne incognita is a sedentary endoparasitic nematode, with total penetration and gall-forming; it has a wide geographical distribution and causes significant agricultural losses. Recently, organic fertilizers and compounds such as chitin have been used in the suppression of plant pests and diseases. The aim of this study was to evaluate the effect of applying cattle manure vermicompost, and its enrichment with chitin, on the infection of $M$. incognita in tomato plants var. Hayslip under greenhouse conditions. For this purpose, plants were inoculated with 5000 units of eggs+second stage juveniles per pot and were compared with controls grown in soil without organic amendment or chitin. The evaluation was carried out 60 days afterwards: the shoot and root fresh weight, shoot dry weight, total population in root and soil nematodes, root knot index (INR), rate of egg masses (IMH), the nematode reproduction factor (FR) and functional-groups
\end{abstract}

** Centro de Investigación en Protección de Cultivos, Universidad de Costa Rica. San José, Costa Rica. 
(hongos, bacterias y actinomicetes). La adición al suelo de vermicompost provocó un incremento en las variables peso fresco y seco foliar y peso fresco radical: a mayor porcentaje de vermicompost, mayor incremento; así mismo, la aplicación del vermicompost redujo la cantidad del nematodo en raíz y en suelo. La aplicación de quitina acentuó estos resultados, obteniéndose el menor factor de reproducción de $M$. Incognita, con un valor de 3,76 para la dosis de $50 \%$ vermicompost+quitina, mientras que en el testigo este factor fue de 93,20. La aplicación de quitina como enmienda única solo afectó la población de hongos en el suelo, mientras que la de actinomicetes aumentó en los tratamientos enmendados.

\section{INTRODUCCIÓN}

Los nematodos son los organismos multicelulares más numerosos de la tierra; la mayoría son de vida libre, otros son parásitos de plantas y pueden cobrar importancia económica en la producción agrícola, en algunos casos con pérdidas superiores al $80 \%$ en zonas con alta infestación (Dong y Zhang 2006, Guerena 2006, Pakeerathan et al. 2009, Raaijmakers et al. 2009).

Meloidogyne es un endoparásito de penetración total y sedentario, formador de nódulos radicales y tiene una amplia distribución geográfica. Se han descrito más de 90 especies, entre ellas, M. arenaria, M. javanica, M. hapla y $M$. incognita relacionadas con importantes pérdidas económicas (De Jin et al. 2005, Rodríguez et al. 2007). Meloidogyne sp., es uno de los patógenos más importantes del cultivo del tomate y limita su producción a nivel mundial, con pérdidas estimadas entre 28 a $68 \%$ (Pakeerathan et al. 2009).

Durante las últimas décadas el control de los nematodos se ha realizado por medio de nematicidas y fumigantes de suelo como el bromuro de metilo. Sin embargo, estos productos numbers (fungi, bacteria and actinomycetes) were measured. The addition of vermicompost to soil caused an increase in the variables of shoot fresh and dry weight and fresh root weight: the higher the percentage of vermicompost, the higher the increase; likewise, the application of vermicompost reduced the number of nematodes both in root and soil. The application of chitin accentuated these results by bringing about the lowest reproduction factor of $M$. incognita, with a value of 3.76 for the dose of vermicompost $+50 \%$ chitin, whereas in the control this factor had a value of 93.20. The application of chitin as sole amendment affected only the soil fungi population, while the actinomycetes population increased in the amended treatments.

resultan inefectivos cuando las poblaciones de nematodos son altas, además, pueden llegar a generar resistencia, afectan la salud humana y el medio ambiente, reducen la biodiversidad de los ecosistemas y por su costo, son frecuentemente inaccesibles para pequeños agricultores (Akhtar y Malik 2000, Pakeerathan et al. 2009).

En la actualidad, existe una enorme presión para limitar su uso, ya que cada vez son más los consumidores que demandan alimentos libres de agroquímicos y se generan políticas internacionales para reducir las fuentes de contaminación ambiental (Kerry 1990, Akhtar y Malik 2000, Oka y Yermiyahu 2002, De Jin et al. 2005, Pakeerathan et al. 2009, Oka 2010). El desarrollo de estrategias de control más amigables con el ambiente, como la rotación de cultivos, el barbecho, los cultivares resistentes, el tratamiento térmico, el control biológico y la aplicación de enmiendas orgánicas son alternativas que deben ser consideradas (Barker y Koenning 1998, Abawi y Widmer 2000, Oka y Yermiyahu 2002).

La adición de enmiendas orgánicas tiene el potencial de modificar la estructura del suelo e influir sobre las poblaciones microbianas 
(Hoitink y Boehm 1999, Oka 2010, Treonis et al. 2010). Entre las enmiendas más comúnmente utilizadas se encuentran los cultivos de cobertura, la adición de abonos verdes y los abonos orgánicos como el compost y el vermicompost (Treonis et al. 2010).

Los mecanismos involucrados en la supresión de nematodos con el uso de enmiendas orgánicas son: 1. Modificación en las propiedades químicas y físicas del suelo. 2. Liberación de compuestos nematicidas preexistentes en el material original. 3. Generación de compuestos nematicidas durante la degradación de los materiales. 4. Incremento en la tolerancia y resistencia de las plantas. 5. Aumento y/o introducción de microorganismos antagonistas. El efecto supresivo de las enmiendas orgánicas sobre los nematodos se debe posiblemente a la combinación de estos mecanismos (Otobe et al. 2004, Treonis et al. 2010, Oka 2010).

Características físicas como la agregación de las partículas de suelo y el tamaño de los poros, se ven afectadas por la adición de abonos orgánicos (Fujimoto et al. 2010, Oka 2010). Otobe et al. (2004) encontraron que suelos con poros de tamaño fino y continuos reducen la movilidad de los nematodos. Otros factores que afectan las poblaciones de esos microorganismos son el $\mathrm{pH}$, las concentraciones de $\mathrm{CO}_{2}$ y oxígeno, la salinidad y el potencial redox (Oka 2010).

La liberación de compuestos volátiles y no volátiles como glucosinolatos, ácidos orgánicos, compuestos nitrogenados $\left(\mathrm{NH}_{3}\right)$ y azufrosos a partir de la descomposición de residuos orgánicos, han mostrado propiedades nematicidas (Raviv et al. 2005, Zasada 2005, Cayuela et al. 2008, Meyer et al. 2009). Abawi y Widmer (2000) indicaron que durante la descomposición de los residuos orgánicos se producen sustancias tóxicas volátiles y no volátiles que inhiben los nematodos parásitos de plantas tales como cianuro de hidrógeno, nitrilos e isotiocianatos.

En relación con la tolerancia o resistencia de las plantas a los nematodos fitoparásitos, Oka (2010) cita 2 causas posibles, el desarrollo de poblaciones de microorganismos inductores específicos y la exposición a compuestos inductores presentes en la enmienda.

Las enmiendas orgánicas pueden introducir microorganismos al suelo o bien aumentar la microflora existente (Hoitink et al. 1997, Treonis et al. 2010), lo que puede afectar negativamente las poblaciones de nematodos ya sea por depredación, parasitismo y competencia por sitios de infección o alimentación sobre la raíz de la planta (Oka 2010, Akhtar 2000).

Sun et al. (2006) estudiaron la microflora asociada a huevos y hembras de Meloidogyne spp., y encontraron que los hongos predominantes fueron Paecilomyces lilacinus, Fusarium spp., Pochonia chlamydosporia, Penicillium spp., Aspergillus spp., Acremonium spp., además de actinomicetes. En ensayos de invernadero, algunos de ellos redujeron el índice de agallamiento hasta en un $60 \%$. Jonathan et al. (2000) estudiaron en tomate y banano el efecto sobre $M$. incognita de las bacterias Bacillus cereus, $B$. subtilis, B. sphaericus, Agrobacterium radiobacter, Pseudomonas fluorescens, P. chlororaphis, Burkholderia cepacia, Pasteuria penetrans, y actinomicetes, todas favorecieron el crecimiento de ambos cultivos y en tomate suprimieron el desarrollo de agallas en las raíces.

Dos tipos de insumos orgánicos que han demostrado potencial en el control de nematodos son los abonos orgánicos y la quitina (Cohen 2001, Oka y Yermiyahu 2002, Merzendorfer y Zimoch 2003, Arancon et al. 2004, De Jin et al. 2005, Gortari y Hours 2008, Oka 2010). Marull et al. 1997 determinaron un número menor de individuos de Meloidogyne javanica en raíces de chile y tomate cultivados en suelos enmendados con compost elaborado con base en residuos municipales. McSorley y Gallaher (1995) encontraron que el uso de compost con base en residuos vegetales aplicados al suelo, redujo las poblaciones de Paratrichodorus minor, Criconemella spp., y Pratylenchus spp., e incrementó la producción de maíz. En tomate Oka y Yermiyahu (2002) encontraron que la adición de compost a base de estiércol bovino, en dosis de 10 o $25 \%$ (v/v), suprimió la presencia de agallas de $M$. javanica. 
Serfoji et al. 2010 indican que la utilización de vermicompost aumentó la producción de tomate, ya que mejoró el contenido de nutrientes en el suelo y la asimilación de los mismos por las plantas, además observaron una reducción en las poblaciones de Meloidogyne incognita. Artavia et al. (2010), Noble y Coventry (2005) y Arancon et al. (2004) informan que la aplicación de vermicompost logró suprimir enfermedades causadas por Phytophthora, Fusarium, Plasmodiophora, Pythium, Rhizoctonia y Verticillium.

Arancon et al. (2004) encontraron que suelos de parcelas tratadas con vermicompost presentaron menos poblaciones de fitonematodos que los tratados con fertilizantes inorgánicos; además las poblaciones de nematodos fungívoros y bacteriófagos fueron mayores en las parcelas tratadas con vermicompost.

Por otro lado la quitina, debido a sus propiedades físico-químicas y biológicas, genera gran interés en el área agrícola (Tharanathan y Kittur 2003, Gortari y Hours 2008), este compuesto es un biopolímero aminado y componente estructural que contribuye a la resistencia mecánica de los organismos que la contienen, después de la celulosa es el polisacárido más abundante en la naturaleza y el principal componente del exoesqueleto de insectos y crustáceos, además forma parte de las paredes celulares de los hongos, algas, y de la estructura básica de los huevos de los nematodos (Cohen 2001, Oka y Yermiyahu 2002, Merzendorfer y Zimoch 2003, De Jin et al. 2005, Gortari y Hours 2008). Debido a que no se encuentra presente en plantas y vertebrados, se puede utilizar de manera segura en el control de plagas y enfermedades (Cohen 2001). La adición de quitina al suelo provoca un resultado adverso sobre las poblaciones de nematodos (Oka y Yermiyahu 2002, De Jin et al. 2005), posiblemente como consecuencia de la producción de compuestos nitrogenados tóxicos como el amoniaco y el ácido nitroso (Oka 2010), y a su capacidad para incrementar la actividad de microorganismos quitinolíticos capaces de degradar la quitina presente en los huevos (Shubakov y Kucheryavykh 2004, De Jin et al. 2005, Nguyen et at. 2007, Gortari y Hours 2008). Los hongos Humicola, Gliocladium, Phoma, Fusarium, Chaetomium, Cylindrocladium, Aspergillus, Penicillium, Trichoderma, Paecilomyces, Sporotrichum, Beauveria, y Mucor, y bacterias como Serratia, Pseudomonas y Burkholderia se citan como microorganismos quitinolíticos (Shubakov y Kucheryavykh 2004, De Jin et al. 2005, Gortari y Hours 2008, Rodríguez-Kabana 1991).

La presente investigación tuvo como objetivo evaluar el efecto de un vermicompost y su enriquecimiento con quitina sobre la infección de Meloidogyne incognita en tomate bajo condiciones de invernadero.

\section{MATERIALES Y MÉTODOS}

La investigación se desarrolló en los invernaderos del Centro de Investigaciones Agronómicas (CIA) y en el Laboratorio de Nematología del Centro de Investigaciones en Protección de Cultivos (CIPROC) de la Universidad de Costa Rica, durante marzo-agosto del 2009.

Incremento de la población de nematodos: Macetas plásticas con $2000 \mathrm{~cm}^{3}$ de suelo tratado previamente con vapor por $24 \mathrm{~h}$, se utilizaron para sembrar plantas de Solanum lycopersicum var., Hyslip. En cada maceta se sembró una planta de tomate y se adicionó una suspensión de huevos y juveniles en segundo estado de Meloidogyne incognita, las plantas inoculadas se mantuvieron en crecimiento por 75 días en invernadero.

Material vegetal para el ensayo: Semillas de tomate var., Hyslip fueron puestas a germinar en bandejas con suelo previamente tratado con calor. Tres semanas después, las plantas estaban listas para ser trasplantadas a macetas plásticas de $2000 \mathrm{~cm}^{3}$ de capacidad con su respectivo tratamiento.

Preparación del suelo y enmiendas orgánicas: El suelo provino de Chachagua de San Carlos (Cuadro 1), se trató con vapor a $96^{\circ} \mathrm{C}$ por $24 \mathrm{~h}$. El vermicompost se obtuvo a base de 
Cuadro 1. Análisis químico del suelo.

\begin{tabular}{cccccccccccccc}
\hline $\mathrm{pH}$ & \multicolumn{1}{c}{} & \multicolumn{3}{c}{$\mathrm{cmol}(+) . \mathrm{l}^{-1}$} & \multicolumn{3}{c}{$\%$} & \multicolumn{3}{c}{$\mathrm{Mg} . \mathrm{l}^{-1}$} & \multicolumn{3}{c}{$\%$} \\
\hline $\mathrm{H}_{2} \mathrm{O}$ & ACIDEZ & $\mathrm{Ca}$ & $\mathrm{Mg}$ & $\mathrm{K}$ & $\mathrm{CICE}$ & $\mathrm{SA}$ & $\mathrm{P}$ & $\mathrm{Zn}$ & $\mathrm{Cu}$ & $\mathrm{Fe}$ & $\mathrm{Mn}$ & $\mathrm{MO}$ \\
6,5 & 0,15 & 4,53 & 0,89 & 0,01 & 5,58 & 3 & $\mathrm{ND}$ & 1,6 & 8 & 54 & 14 & 1,9 \\
\hline
\end{tabular}

estiércol bovino procedente del módulo lechero de la Sede del Atlántico de la Universidad de Costa Rica. La quitina utilizada $\left(\mathrm{C}_{8} \mathrm{H}_{13} \mathrm{NO}_{5}\right)_{\mathrm{n}}$ fue de Sigma-Aldrich (Shirm Shell, practical grade). Se realizó una mezcla de vermicompost (sin tratamiento térmico) y suelo en proporción $25: 75 \%$ y 50:50\% respectivamente, además se preparó una mezcla de suelo con $0,4 \%(\mathrm{p} / \mathrm{p})$ de quitina y las mezclas suelo/vermicompost con quitina en la misma proporción, para llenar las macetas plásticas de $2000 \mathrm{~cm}^{3}$ de capacidad. Tres días después de la preparación de las mezclas, una planta de tomate de 3 semanas fue trasplantada a cada maceta.

\section{Extracción de inóculo de Meloidogyne} incognita: De las plantas de tomate inoculadas se extrajo el sistema radical, se lavó y se procedió a seleccionar las masas de huevos con la ayuda de equipo de disección y un estereoscopio marca Bausch \& Lomb a 45X. Las masas de huevos fueron colocadas en un beaker con agua destilada y un inyector de burbujas de aire por $24 \mathrm{~h}$. Los huevos y juveniles en segundo estado recién eclosionados fueron recuperados con la ayuda de 2 cribas de 0,150 y $0,025 \mathrm{~mm}\left(\mathrm{~N}^{\circ} 100\right.$ y 500 mesh). El contenido de la criba de $0,025 \mathrm{~mm}$ se recolectó en un beaker, se aforó hasta $500 \mathrm{ml}$ y se homogenizó para tomar 10 alícuotas de $5 \mathrm{ml}$ cada una y cuantificar la población.

Inoculación de plantas: Siete días después del trasplante, se realizó la inoculación de M. incognita (Figura 1), alrededor de cada planta y aproximadamente a $1 \mathrm{~cm}$ del tallo se agregaron 5000 unidades de inóculo (huevos+juveniles en segundo estado). Las plantas mantuvieron su crecimiento en condiciones de capacidad de campo, en el invernadero del CIA por 60 días después de la inoculación, no se aplicó fertilización química.

Diseño experimental: Se utilizó un diseño experimental irrestricto al azar con 6 tratamientos y 4 repeticiones. Los tratamientos fueron:

1. $100 \%$ Suelo+M. incognita $(0 \% \mathrm{~V})$.

2. $75 \%$ Suelo $+25 \%(\mathrm{v} / \mathrm{v})$ de vermicompost $+M$. incognita $(25 \% \mathrm{~V})$.

3. $50 \%$ Suelo $+50 \%(\mathrm{v} / \mathrm{v})$ de vermicompost $+M$. incognita $(50 \% \mathrm{~V})$.

4. $100 \%$ Suelo $+0,4 \%(\mathrm{p} / \mathrm{p})$ de quitina $+M$. incognita $(0 \% \mathrm{~V}+\mathrm{Q})$.

5. $75 \%$ Suelo $+25 \% \quad(\mathrm{v} / \mathrm{v})$ de vermicompost $+0,4 \%(\mathrm{p} / \mathrm{p})$ de quitina $+M$. incognita $(25 \% \mathrm{~V}+\mathrm{Q})$.

6. $50 \%$ Suelo+50\% (v/v) de vermicompost $+0,4 \%(\mathrm{p} / \mathrm{p})$ de quitina+M. incognita $(50 \% \mathrm{~V}+\mathrm{Q})$.

Variables evaluadas: Las plantas fueron evaluadas a los 60 días después de la inoculación con $M$. incognita. Cada planta fue cortada en la base, para obtener el peso fresco aéreo y el peso seco aéreo $\left(60^{\circ} \mathrm{C}\right.$ hasta peso constante). En cada repetición previamente identificada, se recuperó el sistema radical y el suelo, para determinar la población final de $M$. incognita. El contenido de la maceta de $2000 \mathrm{~cm}^{3}$ se introdujo en una bolsa plástica, se homogenizó por agitación, se extrajo una muestra de $100 \mathrm{~cm}^{3}$ y se procesó por el método de centrifugación con solución azucarada. El sistema radical fue lavado para obtener el peso fresco, el Índice de Nódulos Radicales (INR) (escala $1=0,2=1$ a $25,3=26$ a $50,4=51$ a 75 y $5=76$ a $100 \%$ del sistema radical agallado) y se realizó una tinción con Floxina-B para determinar el 


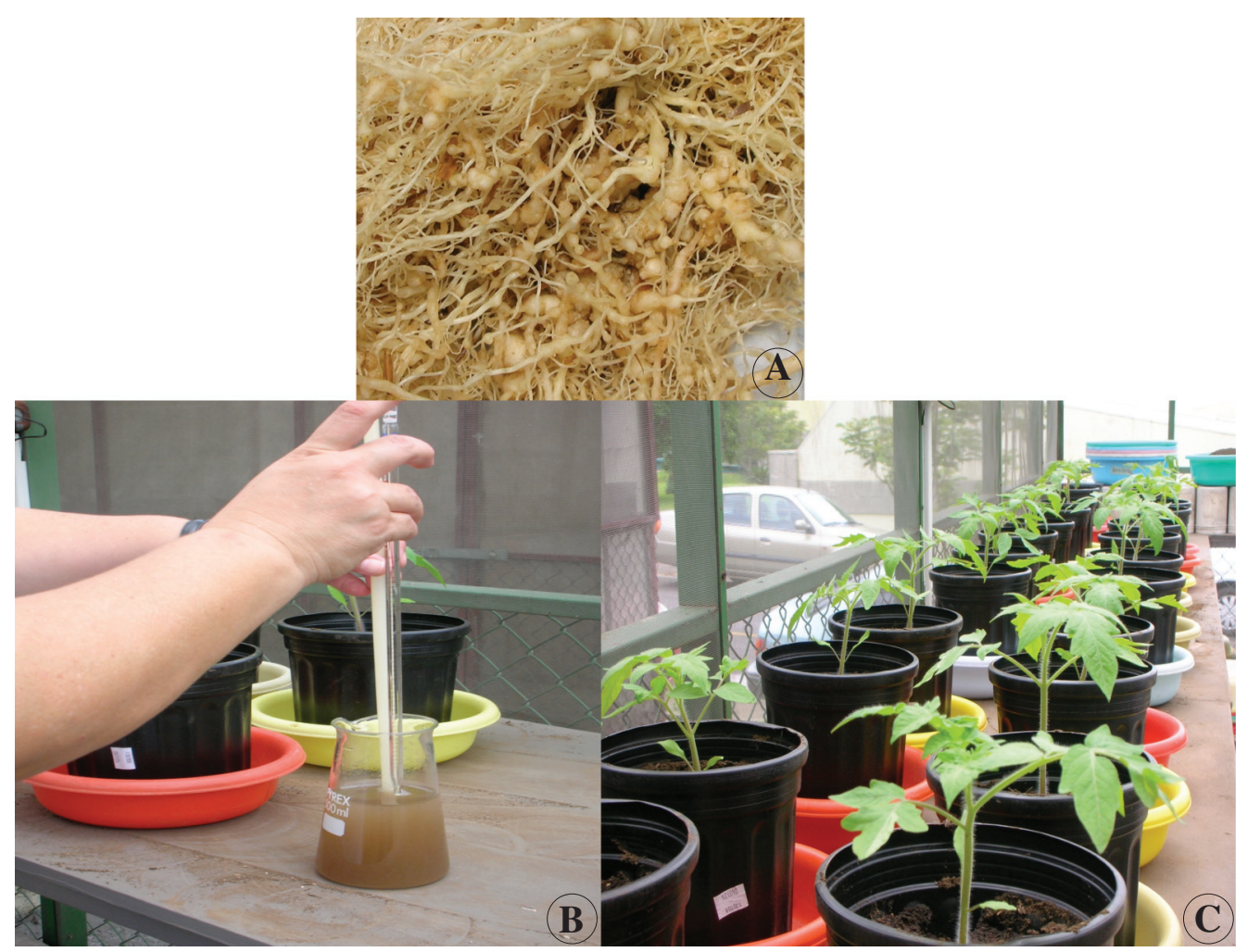

Fig. 1. Inoculación del nematodo. A. Sistema radical de tomate donde se incrementó el inóculo de Meloidogyne incognita para la investigación (edad 75 días). B. Suspensión de nematodos (huevos+J2) utilizada en la investigación. C. Plantas de tomate var. Hyslip, 7 días después del trasplante, preparadas para la aplicación de los nematodos.

Índice de Masas de Huevos (IMH) (escala $0=0$, $1=1$ a $2,2=3$ a $10,4=31$ a 100 y $5=$ más de 100 masas de huevos). Posteriormente cada sistema radical se cortó en trozos de aproximadamente 1 $\mathrm{cm}$ y se procesó con hipoclorito de sodio al $1 \%$ para obtener la población (huevos y juveniles en segundo estado) por medio de cribas de $0,150 \mathrm{y}$ $0,025 \mathrm{~mm}$ ( $\mathrm{N}^{\mathrm{o}} 100$ y $\left.500 \mathrm{mesh}\right)$. Se obtuvo además el Factor de Reproducción (FR=Población final /Población inicial) de M. incognita.

Los conteos de hongos, bacterias, actinomicetes se realizaron por medio de recuentos en platos, para ello se hicieron en cámara de transferencia, diluciones seriadas $(1 / 10)$ del suelo hasta la dilución $1 \times 10^{8}$, a partir de las diferentes diluciones se transfirieron por duplicado alícuotas de 100 ul de cada dilución a platos de albuminato de sodio para el recuento de bacterias y actinomicetes y de Martin para el recuento de hongos (Wollum 1982, Lorch et al. 1995). La evaluación de los platos y conteo de las unidades formadoras de colonias se realizó 5 días después.

Análisis estadístico: Los datos se analizaron por medio del análisis de varianza, como separador de medias, se utilizó la prueba LSD Fisher $(\mathrm{p}=0,05)$. En el caso de los grupos funcionales los datos fueron trasformados con el $\log _{10}$. Se evaluó la normalidad con la prueba de Shaphiro Wilks. 


\section{RESULTADOS Y DISCUSIÓN}

La adición de vermicompost al suelo y en combinación con quitina provocó un incremento en las variables de crecimiento de la planta (Cuadro 2). El peso fresco foliar se incrementó 20 veces cuando se adicionó vermicompost al $25 \%$ y 35 veces con la dosis de $50 \%$, ambos con respecto al tratamiento con solo suelo $(0 \% \mathrm{~V})$, lo que indica que el abono brinda un aporte significativo en la producción de biomasa del cultivo, al respecto Zaller (2007) encontró que la adición de vermicompost llenó los requerimientos nutricionales de plántulas de tomate en vivero. $\mathrm{Al}$ aplicar quitina junto con el vermicompost, el peso fresco se incrementó 30 y 40 veces cuando se utilizó vermicompost al $25 \%$ y a $50 \%$ respectivamente (Cuadro 2). La quitina, material rico en nitrógeno, podría favorecer por medio de la activación de la biomasa microbiana, la producción de sustancias promotoras de crecimiento o la liberación de nutrientes propios o presentes en el abono y contribuir así al crecimiento de la planta. Las dosis vermicompost $25 \%(25 \% \mathrm{~V})$, vermicompost $25 \%+$ quitina $(25 \% \mathrm{~V}+\mathrm{Q})$, vermicompost $50 \%(50 \% \mathrm{~V})$ y vermicompost $50 \%+$ quitina $(50 \% \mathrm{~V}+\mathrm{Q})$ incrementaron significativamente el peso seco foliar con respecto a los tratamientos $0 \% \mathrm{~V}$ y solo suelo+quitina $(0 \% \mathrm{~V}+\mathrm{Q})$. Los resultados referentes al peso fresco de raíz demuestran un incremento significativo con la aplicación del vermicompost con respecto al tratamiento $0 \% \mathrm{~V}$, así, al $25 \% \mathrm{~V}$, se observó un aumento en el peso fresco de raíz de 7 veces, mientras que para la dosis de $50 \% \mathrm{~V}$ el incremento fue de 10 veces. En este sentido, Zaller 2007 observó en plantas de tomate, un aumento en el crecimiento radical de la planta al adicionar vermicompost. El mismo comportamiento se observó al colocar la quitina junto con el vermicompost, el peso fresco radical fue significativamente mayor, en la dosis de $25 \%$ $\mathrm{V}+\mathrm{Q}$ y en la de $50 \% \mathrm{~V}+\mathrm{Q}$ (10 y 15 veces mayor respectivamente en comparación con $0 \% \mathrm{~V}$ ).

Cuando se aplicó vermicompost al 25 y al $50 \%$ y en mezcla con quitina, la densidad poblacional de Meloidogyne incognita en el sistema radical disminuyó de manera significativa respecto al tratamiento $0 \% \mathrm{~V}$, el mismo efecto se presentó en el suelo (Cuadro 3). Al respecto, Khalilian et al. (2002) encontraron que la adición de compost a base de desechos municipales disminuyó la densidad de Hoplolaimus columbus durante los 4 años de estudio, las parcelas tratadas presentaron densidades comparables a las encontradas cuando se utilizó Aldicarb 15G. Serfoji et al. (2010) informaron de una reducción en el número de masas de huevos, en el número de agallas y en el factor de reproducción de Meloidogyne incognita en tomate, luego de aplicar vermicompost junto con Glomus aggregatum y Bacillus coagulans. Khalilian et al. (2002) atribuyen la disminución de la población de nematodos a un aumento en

Cuadro 2. Efecto de vermicompost y la adición de quitina sobre variables de crecimiento.

\begin{tabular}{cccc}
\hline Tratamiento & Peso fresco foliar $(\mathrm{g})$ & Peso seco foliar $(\mathrm{g})$ & Peso fresco radical $(\mathrm{g})$ \\
\hline Suelo & $11,08 \mathrm{a}$ & $1,40 \mathrm{a}$ & $6,11 \mathrm{a}$ \\
$25 \% \mathrm{VE}$ & $216,86 \mathrm{~b}$ & $42,43 \mathrm{~b}$ & $44,43 \mathrm{~b}$ \\
$50 \% \mathrm{VE}$ & $391,10 \mathrm{~cd}$ & $53,05 \mathrm{~b}$ & $63,93 \mathrm{c}$ \\
Suelo+Q* & $11,10 \mathrm{a}$ & $1,27 \mathrm{a}$ & $4,43 \mathrm{a}$ \\
$25 \%$ VE+Q & $330,78 \mathrm{c}$ & $53,15 \mathrm{~b}$ & $61,78 \mathrm{c}$ \\
$50 \%$ VE+Q & $447,62 \mathrm{~d}$ & $59,33 \mathrm{~b}$ & $94,10 \mathrm{~d}$ \\
\hline
\end{tabular}

Cada valor tiene un promedio de 4 repeticiones. Valores seguidos por la misma letra en una misma columna no difieren significativamente cuando el promedio fue separado por diferencia mínima significativa $(\mathrm{p} \leq 0,05)$. 
Cuadro 3. Efecto del vermicompost y la quitina sobre la infección de $M$. incognita en plantas de tomate 2 meses después de la inoculación. ${ }^{v}$

\begin{tabular}{cccccc}
\hline Tratamiento & Meloidogyne I sistema radical & Meloidogyne en suelow $^{\mathrm{w}}$ & $\mathrm{FR}^{\mathrm{x}}$ & INR $^{\mathrm{y}}$ & IMH $^{\mathrm{z}}$ \\
\hline Suelo $(0 \% \mathrm{~V})$ & $408875 \mathrm{~d}$ & $57100 \mathrm{c}$ & $93,20 \mathrm{c}$ & $5,00 \mathrm{~b}$ & $5,00 \mathrm{c}$ \\
$25 \% \mathrm{~V}$ & $176212 \mathrm{bc}$ & $18400 \mathrm{~b}$ & $38,92 \mathrm{~b}$ & $2,75 \mathrm{a}$ & $2,80 \mathrm{a}$ \\
$50 \% \mathrm{~V}$ & $52538 \mathrm{ab}$ & $2900 \mathrm{a}$ & $11,09 \mathrm{ab}$ & $2,75 \mathrm{a}$ & $2,80 \mathrm{a}$ \\
Suelo+Q & $288938 \mathrm{~cd}$ & $53900 \mathrm{c}$ & $68,57 \mathrm{c}$ & $5,00 \mathrm{~b}$ & $4,80 \mathrm{bc}$ \\
$25 \% \mathrm{~V}+\mathrm{Q}$ & $88575 \mathrm{ab}$ & $2100 \mathrm{a}$ & $18,14 \mathrm{ab}$ & $2,50 \mathrm{a}$ & $3,80 \mathrm{ab}$ \\
$50 \% \mathrm{~V}+\mathrm{Q}$ & $15488 \mathrm{a}$ & $3300 \mathrm{a}$ & $3,76 \mathrm{a}$ & $2,00 \mathrm{a}$ & $3,00 \mathrm{a}$ \\
\hline
\end{tabular}

v Cada valor tiene un promedio de 4 repeticiones. Valores seguidos por la misma letra en una misma columna no difieren significativamente cuando el promedio fue separado por diferencia mínima significativa $(\mathrm{p} \leq 0,05)$.

w Cada maceta contenía $2000 \mathrm{~cm}^{3}$ de suelo, vermicompost o quitina solo o en mezcla.

x Factor de Reproducción; FR=Población Final/5000 unidades de inóculo.

y Índice de nódulos radicales.

$\mathrm{z}$ Índice de masas de huevos.

la actividad microbiana producto de la adición de abono orgánico. Chen et al. (2000) reportaron una reducción en la severidad del agallamiento de las raíces de lechuga y menor producción de huevos de $M$. hapla con el uso de compost; además Oka y Yermiyahu (2002) mostraron que la aplicación de compost elaborado a partir de estiércol bovino en dosis de 10 y $25 \%(\mathrm{v} / \mathrm{v})$ inhibió la producción de agallas de $M$. javanica en las raíces de tomate, los extractos acuosos del compost presentaron una alta actividad nematicida especialmente sobre los juveniles, los autores relacionaron este efecto supresivo con altas concentraciones de $\mathrm{N}$, especialmente $\mathrm{N}-\mathrm{NH}_{3}$, y una alta conductividad eléctrica de la enmienda.

La aplicación de quitina en mezcla con $50 \%$ de vermicompost, mostró una reducción significativa en el número de $M$. incognita en el sistema radical (15 488 nematodos) con respecto a $0 \% \mathrm{~V}$ y al tratamiento Suelo+Q (408 875 y 288 938 nematodos.sistema radical $^{-1}$ respectivamente) (Cuadro 3).

Con respecto a $M$. incognita en suelo, se observó una disminución significativa en esa variable al aumentar la dosis de vermicompost (18 400 nematodos para $25 \% \mathrm{~V}$ y 2900 nematodos para $50 \% \mathrm{~V}$ ) en relación con el tratamiento 0\%V (57 100 nematodos.2000 $\mathrm{cm}^{-3}$ de suelo). La adición de quitina a la mezcla con vermicompost al 50\% logró reducir drásticamente la población de nematodos en el suelo si se compara con el tratamiento $0 \% \mathrm{~V}$ (3300 y 57100 nematodos respectivamente) (Cuadro 3).

El factor de reproducción (FR) de $M$. incognita se redujo cuando se aplicaron los tratamientos $25 \% \mathrm{~V}, 50 \% \mathrm{~V}, 25 \% \mathrm{~V}+\mathrm{Q}$ y $50 \% \mathrm{~V}+\mathrm{Q}$ (Cuadro 3). El mayor efecto sobre el FR se logró con la adición de $50 \% \mathrm{~V}+\mathrm{Q}(3,76)$, comparado con un FR de 93,2 en el tratamiento control $(0 \% \mathrm{~V})$.

En los tratamientos $0 \% \mathrm{~V}$ y $0 \% \mathrm{~V}+\mathrm{Q}$, se obtuvieron los mayores valores del INR (5 en ambos tratamientos) y del IMH (5 y 4,8 respectivamente), mientras que con la aplicación de vermicompost solo o en mezcla con quitina se presentaron valores entre 2,0 y 3,8 para la variable IMH. De Jin et al. (2005) indicaron que la aplicación de compost con quitina redujo de manera significativa el desarrollo de las agallas en las raíces de plantas de tomate en un 64\% respecto al control. Los autores indican que la actividad quitinolítica derivada de adicionar al suelo el compost con quitina puede ser un factor relevante en la reducción de la infección por nematodos, datos que concuerdan con lo obtenido en esta investigación donde el enriquecimiento de 
vermicompost con quitina logró la mayor reducción de las poblaciones de $M$. incognita.

La aplicación de quitina al suelo como enmienda única provocó cambios significativos sobre la población de hongos, ya que aumentó el número de propágulos (Figura 2). Los hongos han mostrado gran potencial en el control de nematodos, según algunos autores debido a la generación de enzimas extracelulares en las que se incluyen las quitinasas (Akhtar y Malik 2000, Kerry 1990, Sun et al. 2006, Nguyen et al. 2007). Halmman et al. (1999) encontró que la adición de quitina en el suelo al 1\% (p/p) causó la supresión $M$. incognita, en plantas de algodón y relacionó esta supresión con el aumento de las poblaciones de hongos. La adición de vermicompost con y sin quitina no causó cambios significativos en el número de propágulos de hongos con respecto al tratamiento $0 \% \mathrm{~V}$. Por otro lado, al incrementarse la dosis de vermicompost al $50 \%$ se observa una tendencia a una menor población de bacterias (Cuadro 3), que resulta significativa en el caso del tratamiento 50\%VQ. La población de actinomicetes se vio incrementada tanto por la adición de vermicompost como por la adición de quitina (Figura 2). Los actinomicetos son uno de los grupos de bacterias más abundantes (90\%), muestran una alta actividad biodegradativa al secretar un amplio rango de enzimas que les permiten metabolizar sustancias complejas estables (Cardona et al. 2009).

Powlsen et al. (2008) informaron que la adición de quitina al compost provoca cambios en la composición de la comunidad microbiana y Halmman et al. (1999) indican que la supresión de los nematodos parásitos de las plantas debido a la adición de quitina no sólo está asociada con cambios microbianos en el suelo y rizosfera, sino también con cambios en la comunidad de bacterias dentro del tejido. Los mismos autores indican que es posible utilizar abonos orgánicos enmendados con productos como la quitina como una herramienta para el manejo de nematodos patógenos de plantas.

El vermicompost bajo las condiciones evaluadas tuvo un efecto significativo en la reducción de las poblaciones de $M$. incognita en tomate, se encontró además que el efecto se puede acentuar mediante quitina en mezcla con el vermicompost. Es necesario en próximas investigaciones evaluar el efecto del enriquecimiento con residuos que contienen quitina, como

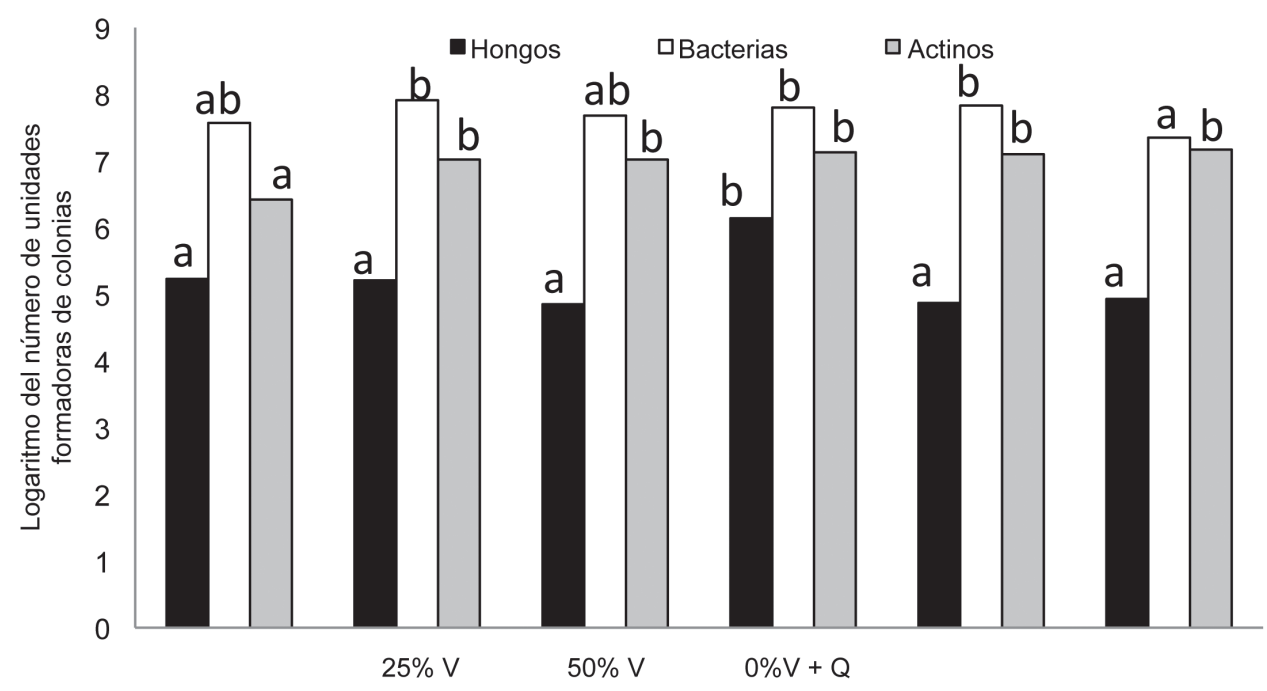

Fig. 2. Efecto de vermicompost y la adición de quitina sobre la población de hongos del suelo. 
los desechos de camarón u otro crustáceo, en la producción de compost o vermicompost, como una alternativa para el control de Meloidogyne incognita y aunado a ello, una vía para reducir los problemas de contaminación asociados a estos residuos.

\section{AGRADECIMIENTOS}

Esta investigación fue realizada con financiamiento de los proyectos VI-733-A8-089 y VI733-A1-821 de la Vicerrectoría de Investigación de la Universidad de Costa Rica.

\section{LITERATURA CITADA}

ABAWI G.S., WIDMER T.L. 2000. Impact of soil health management practices on soilborne pathogens, nematodes and root diseases of vegetable crops. Applied Soil Ecology. 15:37-47.

AKHTAR M., MALIK A. 2000. Roles of organic soil amendments and soil organisms in the biological control of plant-parasitic nematodes: a review. Bioresource Technology 74:35-47.

ARANCON N., GALVIS P., EDWARDS C., YARDIM E. 2004. The trophic diversity of nematode communities in soils treated with vermicompost. Pedobiología. 47(5):736-740.

ARTAVIA S., URIBE L., SABORÍO F., ARAUZ L.F., CASTRO L. 2010. Efecto de la aplicación de abonos orgánicos en la supresión de Pythium myriotylum en plantas de tiquizque (Xanthosoma sagittifolium). Agronomía Costarricense 34(1):17-29.

BARKER K., KOENNING S. 1998. Developing Sustainable Systems for Nematode Management. Annual Review Phytopathology. 36:165-205.

CARDONA G., PEÑA-VENEGAS C., RUIZ-GARCÍA M. 2009. Comunidades de hongos actinomicetos en tres tipos de vegetación de la Amazonia colombiana: abundancia, morfotipos y el gen 16s ADNr. Revista Biología Tropical. 57(4):1119-1139.

CAYUELA M., MILLNER P., MEYER S., ROIGA A. 2008. Potential of olive mill waste and compost as biobased pesticides against weeds, fungi, and nematodes. Science of the total environment. 399:11-18.
CHEN J., ABAWI G., ZUCKERMAN B. 2000. Efficacy of Bacillus thuringiensis, Paecilomyces marquandii, and Streptomyces costaricanus with and without Organic Amendments against Meloidogyne hapla Infecting Lettuce. Journal of Nematology. 32(1):7077.

COHEN E. 2001. Chitin synthesis and inhibition: a revisit. Pest Management Science. 57:946-950.

De JIN R., SUH J., PARK R., KIM Y. 2005. Effect of chitin compost and broth on biological control of Meloidogyne incognita on tomato (Lycopersicon esculentum Mill.). Nematology. Vol. 7(1):125-132.

DONG L., ZHANG Q. 2006. Microbial control of plantparasitic nematodes: a five-party interaction. Plant Soil. 288:31-45.

FUJIMOTO T., HASEGAWA S., OTOBE K., MIZUKUBO T. 2010. The effect of soil water flow and soil properties on the motility of second-stage juveniles of the root-knot nematode (Meloidogyne incognita). Soil Biology \& Biochemistry 42:1065-1072.

GORTARI M., HOURS R. 2008. Fungal chitinases and their biological role in the antagonism onto nematode eggs. A review Mycology Progress. 7:221-238.

GUERENA M. 2006. Nematodes: Alternative Controls. A Publication of ATTRA-National Sustainable Agriculture. $18 \mathrm{pp}$.

HALLMANN J., RODRÍGUEZ-KÁBANA R., KLOEPPER J. 1999. Chitin-mediated changes in bacterial communities of the soil, rhizosphere and within roots of cotton in relation to nematode control. Soil Biology and Biochemistry. 31(4):551-560.

HOITINK H.A.J., STONE A.G., HAN D.Y. 1997. Supresión de enfermedades mediante compost. Agronomía Costarricense. 21(1):25-33.

HOITINK H.A.J., BOEHM M.J. 1999. Biocontrol within the context of soil microbial communities: A substrate-dependent phenomenon. Annual Review Phytopathology. 37:427-446.

JONATHAN E., BARKER K., ABDEL-ALIM F., VRAIN T., DICKSON D. 2000. Biological control of Meloidogyne incognita on tomato and banana with rhizobacteria, actinomycetes, and Pasteuria penetrans. Nematropica 30(2):231-240.

KHALILIAN A., SULLIVAN M., MUELLER J., SHIRALIPOUR A., WOLAK F., WILLLIAMSON R., LIPPERT R. 2002. Effects of surface 
application of MSW compost on cotton productionsoil properties, plant responses and nematode management. Compost Science \& Utilization. 10(3):270-279.

KERRY B. 1990. An Assessment of Progress toward Microbial Control of Plant-parasitic Nematodes. Supplement to Journal of Nematology. 22(4):621-631.

LORCH H.J., BENCKIESER G., OTTOW J.C.G. 1995. Basic methods for counting microorganisms in soil and water, pp. 146-191. In: K. Alef y P. Nannipieri (eds). Methods in applied soil microbiology and biochemistry. Academic Press.

MARULL J., PINOCHET J., RODRÍGUEZ-KABANA R. 1997. Agricultural and municipal compost residues for control of root-knot nematodes in tomato and pepper. Compost Science Utilization. 5:6-15.

MCSORLEY R., GALLAHER R.N. 1995. Effect of yard waste compost on plant parasitic nematode densities in vegetable crops. Journal Nematology. 27:545-549.

MERZENDORFER H., ZIMOCH L. 2003. Chitin metabolism in insects: structure, function and regulation of chitin synthases and chitinases. Journal of Experimental Biology. 206:4393-4412.

MEYER S., RICE C., ZASADA I. 2009. DIBOA: Fate in soil and effects on root-knot nematode egg numbers. Soil Biology \& Biochemistry. 41:1555-1560.

NGUYEN A., KIMA Y., OHA K., JUNGA W., PARKA R. 2007. The role of chitinase from Lecanicillium antillanum B-3 in parasitism to root-knot nematode Meloidogyne incognita eggs. Biocontrol Science and Technology. 10:1047-1058.

NOBLE R., COVENTRY E. 2005. Suppression of soil-borne plant diseases with composts: A review. Biocontrol Science and Technology. 15:3-20.

OKA Y. 2010. Mechanisms of nematode suppression by organic soil amendments. A review. Applied Soil Ecology 44:101-115.

OKA Y., YERMIYAHU U. 2002. Suppressive effects of composts against the root-knot nematode Meloidogyne javanica on tomato. Nematology 4(8):891-898.

OTOBE K., ITOU K., MIZUKUBO T. 2004. Micro-moulded substrates for the analysis of structure-dependent behaviour of nematodes. Nematology. 6:73-77.
PAKEERATHAM K., MIKUNTHAN G., THARSHANI N. 2009. Effect of different animal manures on Meloidogyne incognita (Kafoid and White) on tomato. World Journal of Agricultural Sciences. 5(4):432-435.

POULSEN P., MØLLER J., MAGID L. 2008. Determination of a relationship between chitinase activity and microbial diversity in chitin amended compost. Bioresource Technology. 99:4355-4359.

RAAIJMAKERS J., PAULITZ T., STEINBERG C., ALABOUVETTE C., MOËNNE-LOCCOZ Y. 2009. The rhizosphere: a playground and battlefield for soilborne pathogens and beneficial microorganisms. Plant Soil. 321:341-361.

RAVIV M., OKA Y., KATAN J., HADAR Y., YOGEV A., MEDINA S., KRASNOVOSKY A., ZIADNA H. 2005. High nitrogen compost as a medium for organic cointainer-grown crops. Bioresource Technology. 96:419-427.

RODRÍGUEZ M., GÓMEZ L.Y., PETEIRA B. 2007. Meloidogyne mayaguensis (Rammah y Hirschmann), plaga emergente para la agricultura tropical y subtropical. Revista de Protección Vegetal. 22(3):183-198.

RODRÍGUEZ-KABANA R. 1991. Control biológico de nematodos parásitos de plantas. Nematropica 21(1):111-122.

SERFOJI P., RAJESHKUMAR S., SELVARAJ T. 2010 Management of root-knot nematode, Meloidogyne incognita on tomato cv. Pusa Ruby by using vermicompost, AM fungus, Glomus aggregatum and mycorrhiza helper bacterium, Bacillus coagulans. Journal of Agricultural Technology. 6(1):37-45.

SHUBAKOV A., KUCHERYAVYKH P. 2004. Chitinolytic Activity of Filamentous Fungi. Applied Biochemistry and Microbiology. 40(5):445-447.

SUN M., GAO L., SHI Y., LI B., LIU X. 2006. Fungi and actinomycetes associated with Meloidogyne spp., eggs and females in China and their biocontrol potential. Journal of Invertebrate Pathology. 93(1):22-28.

TERMORSHUIZEN A.J., VAN RIJN E., VAN DER GAAG D.J., ALABOUVETTE C., CHEN Y., LAGERLÖF J., MALANDRAKIS A.A., PAPLOMATAS E.J., RÄMERT B., RYCKEBOER J., STEINBERG C., ZMORA-NAHUM S. 2006. Suppressiveness of 18 composts against 7 pathosystems: Variability in 
pathogen response. Soil Biology and Biochemistry. 38:2461-2477.

THARANATHAN R., KITTUR F. 2003. Chitin: the undisputed biomolecule of great potential. Critical Review in Food Science. 43(1):61-87.

TREONIS A., AUSTIN E., BUYER J., MAUL J., SPICER L., ZASADA I. 2010. Effects of organic amendment and tillage on soil microorganisms and microfauna. Applied Soil Ecology. 46(1):103-110.

WOLLUM A.G. 1982. Cultural methods for soil microorganisms, pp. 781-802. In: A.L. Page, R.H.
Miller y K.R. Keeney (eds). Methods of soil analysis. Part 2. Chemical and microbiological properties. ASA y SSSA.

ZALLER J.G. 2007. Vermicompost in seedling potting media can affect germination,biomass allocation, yields and fruit quality of three tomato varieties. Scientia Horticulturae. 112:191-199.

ZASADA S., MEYER J., HALBRENDT M., RICE C. 2005. Activity of Hydroxamic Acids from Secale cereale Against the Plant-Parasitic Nematodes Meloidogyne incognita and Xiphinema americanum. Phytopathology. 95 (10):1116-1121. 